\title{
Carnets
}

Revue électronique d'études françaises de l'APEF

Première Série - 2| 2010

L'équivoque

\section{Le traducteur et l'équivoque ou la nécessité de ne pas choisir}

Sophie Léchauguette

\section{(2) OpenEdition}

Journals

Édition électronique

URL : http://journals.openedition.org/carnets/4488

DOI : $10.4000 /$ carnets.4488

ISSN : 1646-7698

Éditeur

APEF

Édition imprimée

Date de publication : 1 janvier 2010

Pagination : 19-33

\section{Référence électronique}

Sophie Léchauguette, «Le traducteur et l'équivoque ou la nécessité de ne pas choisir », Carnets [En ligne], Première Série - 2 | 2010, mis en ligne le 16 juin 2018, consulté le 19 avril 2019. URL : http:// journals.openedition.org/carnets/4488; DOI : 10.4000/carnets.4488

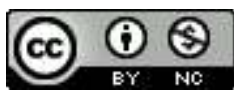

Carnets est mis à disposition selon les termes de la licence Creative Commons - Atribution - Pas d'utilisation commerciale 4.0 International. 


\title{
LE TRADUCTEUR ET L'ÉQUIVOQUE \\ Ou la nécessité de ne pas choisir
}

SOPHIE LECHAUGUETTE

Bordeaux 1- Bordeaux 3 EA4196 CLIMAS

s.lechauguette@omega.u-bordeaux1.fr

\begin{abstract}
Résumé
La réflexion de départ, portant sur la pratique du traducteur, commence par évoquer dans quelles circonstances l'équivoque se présente à lui. Cet examen conduit à s'interroger sur la possibilité d'envisager l'équivoque comme modalité du double. Du fait de langue, on en vient à proposer une définition du terme qui le débarrasse des connotations négatives acquises au cours de l'histoire. Ainsi réhabilitée, l'équivoque devient potentiellement le lieu où peuvent co-exister à égalité deux voix : celles des deux cultures en présence lors de la traduction-activité puis dans la traduction-texte, dispensant son auteur du choix entre position sourcière ou cibliste. II est alors libre de les laisser s'exprimer à égalité.
\end{abstract}

\section{Abstract}

From looking at the way translators face the problem of equivoque in their professional activity, this essay moves on to probing the notion itself moving from the idea of duality to that of doubleness. Soon the encounter of two languages at work through translation leads to offering a definition of equivoque which redeems the term from the negative connotations inherited from history. Thus rehabilitated, an equivoque becomes a place where potentially two cultures are able to coexist. It follows that the translator, freed from the false necessity to stand either on the side of the source text or the target text, becomes free to produce a text giving an equal voice to both.

Mots-clés: traduction, équivoque, source, cible Keywords: translation, equivoque, source, target 
L'équivoque admirable (la primorosa equivocacion) est comme une parole à deux tranchants et une signification à deux lumières. Son artifice consiste à user de quelque mot qui ait deux significations, de manière à créer le doute sur ce que l'on a voulu dire.

Jean-Pierre Cavaillé

Diversement appréciée lorsqu'elle survient entre locuteurs d'une même langue, l'équivoque est instinctivement perçue comme un piège dangereux pouvant se refermer sur le traducteur qui agit au contact de deux langues. Pourtant ce concept ne pourrait-il s'avérer utile et apporter un nouvel éclairage à la réflexion en traductologie, discipline qui s'intéresse à la fois à l'activité traduisante et au devenir de la circulation des textes traduits sur les rapports entre les cultures ? Partant de l'usage et de la définition de l'adjectif, puis du substantif, l'évolution du regard porté sur l'équivoque sera brièvement évoquée. Ces préliminaires amènent à élargir, puis redéfinir la notion dans le cadre de la traduction. L'équivoque créée dans et par le discours, par celui qui en est à l'origine, amène à en envisager une autre : celle résultant de l'absence de références communes entre émetteur et destinataire du discours. Lorsqu'on met en présence des interlocuteurs issus de languescultures différentes, cette absence devient vite manifeste. Choisis parce qu'ils évoqueront probablement des souvenirs à tout lecteur ayant fait l'apprentissage d'une seconde langue puis, à travers elle, découvert une autre culture, quelques-uns des exemples évoqués font apparaître qu'à côté de l'équivoque ancrée dans le linguistique, il en est une ancrée dans l'extralinguistique. II n'y a pas que quand le traducteur est aux prises avec les mots d'un texte - littéraire ou non -, comportant une équivoque, qu'il travaille à son contact. II lui appartient de la déceler, puis d'en déterminer la nature volontaire ou involontaire et enfin de la distinguer de l'ambiguïté pour proposer une traduction capable de jouer son rôle pour des destinataires que l'auteur du texte original n'envisageait pas au départ.

Précisons avant d'aller plus loin que par texte, on entend ici tout type de texte. II ne s'agit pas de se limiter à la littérature car l'équivoque peut surgir dans le discours banal et ordinaire de tout un chacun, par écrit ou dans l'oralité. L'auteur pourra donc être écrivain mais aussi rédacteur, journaliste, syndicaliste...., voire une équipe chargée de produire un texte plus utilitaire qu'esthétique mais aussi un simple locuteur. 


\section{Vers une définition de l'équivoque}

\section{Cerner l'équivoque à travers ses conséquences}

Le terme, on le sait, peut être adjectif ou substantif. Dans son acception la plus usuelle, selon le Trésor de la Langue Française", l'adjectif désigne "ce qui peut revêtir plusieurs significations". La conséquence fâcheuse de cette polysémie est le risque potentiel de faire naître l'incertitude, voire le doute qui génère la méfiance. Appliqué à une personne, une situation, l'usage commun lui donne un sens assez négatif, faisant de lui un équivalent de peu fiable ou non digne de confiance. S'il n'est pas courant de parler de traduction équivoque, on peut dire qu'une traduction fautive, incapable de communiquer à ses lecteurs à la fois le vouloir dire auctorial et la manière de dire, risque de susciter une équivoque au niveau de sa réception. C'est le cas de figure le plus malheureux. L'auteur ainsi traduit, qu'il soit bien ou mal accueilli par la culture destinataire de la traduction, y est sinon incompris, du moins mécompris, ou pris pour ce qu'il n'est pas. On se souvient de la réaction de Milan Kundera qui, découvrant la traduction française de La Plaisanterie ${ }^{2}$, entreprit la révision de tous ses romans publiés dans cette langue, où il ne retrouvait pas son style. L'équivoque, qui dans ce cas, portait sur la perception d'une écriture, a été révélée et levée par l'intervention de l'auteur moins de vingt ans plus tard. Mais de nombreuses équivoques ainsi créées par des traductions, fussent-ce de "belles infidèles", restent longtemps, voire à jamais, invisibles. Combien de lecteurs auront donc aimé ou détesté un traducteur - tout en ignorant les auteurs étrangers qu'ils lisaient, ou croyaient lire puisque la traduction se faisait miroir, reflétant la manière d'écrire de leur propre culture. C'est que le traducteur, à dessein ou peut-être parfois sans réelle intentionalité, a traduit le texte de départ, selon les normes de la culture destinataire.

Double d'un texte premier inaccessible aux lecteurs de traductions, le texte traduit doit non seulement être fiable mais aussi perçu comme tel. Sans quoi, incapable de lui accorder sa confiance, le lecteur déplorerait d'être obligé de s'en remettre au traducteur, ce médiateur qui intervient entre les langues et les cultures. Postulant inconsciemment ou naïvement que le texte de l'auteur relève de la perfection, il déplorerait de n'y avoir pas lui-même directement accès. Insatisfaisante, la traduction engendre la frustration. La communication imparfaite qui s'ensuit est d'autant plus malencontreuse qu'intervenant au moment du passage d'une langue à l'autre, elle peut avoir des conséquences politiques et diplomatiques

\footnotetext{
Accessible en ligne <http://atilf.atilf.fr/tlf.htm>, pour le mot qui nous intéresse $<$ http://atilf.atilf.fr/dendien/scripts/tlfiv5/affart.exe?19;s=1002723750;?b=0;>

2 Gallimard 1968, révisée par l'auteur en 1980 et publié en 1985 avec son commentaire sur la première traduction.
} 
ou, au niveau du polysystème ${ }^{3}$, interdire à des lecteurs d'apprécier les qualités d'écriture d'un écrivain étranger. Un grand auteur peut rester ignoré des lecteurs ne pouvant le lire dans sa langue parce qu'aucun traducteur n'a, jusque là, eu le talent nécessaire pour recréer son œuvre. Mais s'attarder sur les éventuelles conséquences d'une traduction ratée, quand bien même la raison de l'échec serait le non repérage ou une mauvaise traduction des équivoques du texte nous éloignerait de notre sujet. Comparons l'équivoque au calembour et à l'ambiguïté pour en préciser la définition.

\section{Equivoque et calembour}

Apparentée au calembour, l'équivoque joue sur les sonorités des mots, qu'il s'agisse de cas d'homophonie ou de la réunion de plusieurs termes, donnant à entendre un autre mot ou sens, comme dans ces vers de Verlaine où l'oreille peut douter :

Le soir tombait, un soir équivoque d'automne,

Les belles, se pendant, rêveuses, à nos bras,

Dirent alors des mots si spécieux, tout bas

Que notre âme depuis ce temps tremble et s'étonne

La présence du mot équivoque dans le vers précédant celui où le poète joue sur I'homophonie du mot cependant, et de la forme verbale se pendant attire l'attention sur le procédé, témoignant de son art. Dans le poème holorime de Marc Monnier, souvent attribué à Victor Hugo, que l'on connaît rarement en entier mais dont presque tout le monde peut citer ces deux vers

Gall, amant de la reine, à la Tour Magne à Nîmes

Galamment de l'arène, alla tour magnanime.

c'est la multiplication de l'usage du procédé qui alerte le lecteur. L'équivoque est ici synonyme de calembour, cas où, comme le rappelle Messiaen ${ }^{4}$, le jeu porte sur les sonorités indépendamment de la limite que constitue le mot.

Ces équivoques volontaires basées sur l'homophonie ou la polysémie, dont le ressort réside dans le linguistique, sont facilement identifiables par le traducteur. Les problèmes de traduction qu'elles posent, d'ordre lexical, seront résolus avec des stratégies similaires à celles mises à l'œuvre pour la traduction des jeux de mots. II s'agit, plus qu'en aucun autre cas, de re-création ${ }^{5}$. Pour retrouver l'effet produit dans une autre langue, le traducteur doit

\footnotetext{
${ }^{3}$ L'école de Tel Aviv dont les représentants les plus connus sont Even-Zohar et Gideon Toury s'intéresse aux interactions entre littératures en traduction et littératures en VO.

${ }^{4}$ Auteur d'un article où, cherchant à distinguer clairement la syllepse du calembour, il commence par analyser la définition de l'équivoque fournie par le TLF.

${ }^{5}$ On se reportera au remarquable ouvrage de Jacqueline Henry sur le sujet.
} 
être capable de s'affranchir du fétichisme du sémantisme ${ }^{6}$. Fort heureusement, la pluralité de sens se limite souvent au double sens qu'il est parfois possible de conserver au-delà de la barrière des langues. II suffit de penser au terme traduction: ne renvoie-t-il pas, dans plusieurs langues européennes, au moins, autant à l'action de traduire qu'au produit fini en résultant? Quand la langue d'arrivée ne dispose d'aucun terme permettant de jouer sur deux plans à la fois, le traducteur doit savoir se montrer créatif. Sa tâche sera moins ardue quand l'équivoque relève de la situation. Le double sens résulte d'un décalage de connaissance des faits (réels ou fictifs) par deux destinataires distincts (voir étude de cas infra). Les équivoques ainsi créées, dont le ressort se trouve dans l'extra-linguistique, ne sont pas nécessairement volontaires puisqu'elles résultent de la confrontation de deux expériences différentes du monde. L'équivoque ne surgit plus d'une manipulation habile pratiquée sur une langue parfaitement maîtrisée mais des divergences entre les présupposés de l'émetteur du discours et ceux de son récepteur. La différence culturelle suffit à créer des équivoques, même entre locuteurs d'une même langue. Quand elle s'ajoute au fait de parler des idiomes différents, l'intervention du médiateur interculturel qu'est le traducteur va permettre de lever l'équivoque involontaire qui sinon risque fort de passer inaperçue, avec des conséquences parfois fâcheuses comme on va le voir. La traduction est un cas emblématique, mais pas unique, réunissant deux "interlocuteurs" qui ne partagent pas le même bagage cognitif. Par conséquent, comme l'exemple suivant le rappelle, le récepteur peut recevoir les propos de l'émetteur d'une manière qu'il n'avait pas anticipée dans le processus d'encodage.

On entend parfois dire que les discours scientifiques et technique s'affranchissent de la dimension culturelle puisque, à la différence du discours littéraire, ils ont une valeur uniquement référentielle et non esthétique.

... on a affaire d'un point de vue linguistique à un signifié qui devra être le même dans toutes les langues qui l'exprimeront. La description d'un moteur d'avion contient un signifié qui est exactement le même dans toutes les langues et ne peut varier au gré des cultures. (Bocquet, $2007: 13)$

II serait en effet difficile de ne pas partager ce point de vue. On se souviendra qu'en 1999, la NASA perdit un appareil envoyé en orbite autour de Mars. Les ingénieurs de Lockheed avaient programmé un logiciel destiné à transmettre des données en pound second, unité britannique. Leurs collègues américains, habitués à travailler avec des Newton, unité métrique, ont lu les chiffres qu'ils recevaient sans soupçonner qu'ils puissent être dans une autre unité. La disparition du signifiant est ici cause de l'équivoque résultant du changement de signifié non détecté. II s'agit donc d'un problème de communication entre

\footnotetext{
$\overline{{ }^{6} \text { L'expression est de Sergio Viaggio. }}$
} 
cultures, peut-être "séparées par une langue commune". Si les ingénieurs en question ne s'étaient pas exprimés en anglais, la médiation de traducteurs en amont, au moment de la définition du cahier des charges, aurait probablement révélé le problème et évité l'accident. L'intervention d'un intermédiaire au fait des présupposés des différents intervenants aurait fait émerger le non-dit constitutif de l'équivoque culturelle. Cette méprise s'est produite parce que les uns et les autres étaient enfermés dans une vision univoque. Même dans les textes à dominante technique, il y a toujours des moments où la présence du culturel brouille la distinction générique et revient au galop. Et d'ailleurs, Bocquet insiste bien sur le fait que le signifié doit être le même mais ne parle pas du signifiant qui peut et doit souvent varier pour renvoyer à un même signifié.

\section{Équivoque et ambiguïté}

Les équivoques ancrées dans l'extra-linguistique, et donc situationnelles ou culturelles posent un problème antérieur à celui de leur traduction: c'est celui de leur détection, condition préalable à leur traitement pour éviter des catastrophes comme celle évoquée précédemment. Pas plus que le texte premier univoque, la traduction ne doit confronter ses lecteurs à une équivoque. C'est alors que se pose le problème de la distinction entre équivoque ou ambiguïté. L'une comme l'autre peuvent apparaître comme des passages manquant de clarté et laisser leurs destinataires - et donc le traducteur - dans le doute. Leur fonctionnement est toutefois très différent. L'ambiguïté, normalement involontaire, est souvent le fruit d'une maladresse d'expression sauf si l'auteur ou interlocuteur, perfide, est volontairement ambigu pour ne pas être compris. Dans les deux cas, l'incompréhension qu'elle suscite révèle l'ambiguïté. La difficulté de compréhension invite le lecteur à s'interroger et le traducteur à clarifier pour ses lecteurs, poursuivant un travail éditorial peutêtre insuffisamment abouti sur le texte de départ. Exception faite des cas où la visée du discours est de susciter l'incompréhension, peut-être pour plonger ses destinataires dans la perplexité, les faire passer pour des idiots et les disqualifier, l'ambiguïté invite le traducteur à revoir le texte de son auteur pour l'améliorer.

Délibérée ou fortuite, l'équivoque fait sens et risque, on l'a vu, de passer inaperçue pour un public ou des lecteurs distraits ou incapables d'y avoir accès parce qu'ils ne partagent pas le bagage cognitif de l'auteur. Le sens unique alors compris n'est pas celui que veut transmettre son émetteur qui, dans le premier cas, joue sur le double sens et dans le second, n'anticipait pas la possibilité de la méprise, conséquence de l'équivoque involontaire. Quel qu'il soit, le message, exprimé au moyen d'une équivoque volontaire, vise à attirer l'attention sur la coexistence de deux possibilités. Que son destinataire passe à côté, il rate l'essentiel du vouloir-dire, ici (mais n'est-ce pas toujours le cas?) indissociable de sa 
forme. II en résulte un malentendu qui se dissipera ou pas. L'équivoque est à traduire, si possible, par une équivoque autrement s'il le faut, pourvu que le texte traduit fasse lui aussi percevoir à son destinataire la possibilité de la dualité.

Quand elle est l'expression d'une intentionnalité, l'équivoque est à rapprocher des figures du discours en ce qu'elle doit être identifiée pour fonctionner. Elle s'en distingue toutefois en ce que "[elle] relève de l'ordre de la logique, du domaine de la pensée, alors que la syllepse est un trope" (Messiaen : 2). Trope, syllepse ou autres sont des procédés rhétoriques dont l'emploi vise à produire un effet sur les destinataires prévus. L'équivoque, telle qu'on en vient à l'envisager, apparaît davantage comme la résultante de moyens mis en œuvre. Celle qui survient de la mise à disposition du texte pour des destinataires seconds ne partageant pas les connaissances prêtées aux destinataires premiers est à traiter comme une ambiguïté - également involontaire - par le traducteur: agissant en tant que médiateurs, il doit avoir toute latitude de traduire ces passages en les réécrivant pour s'assurer de la bonne compréhension du vouloir-dire véhiculé, indépendamment de sa formulation initiale. Pour remplir son contrat, qu'il s'agisse de celui - juridique - passé avec un éditeur ou un employeur, ou le contrat moral passé entre d'une part lui-même et l'auteur et d'autre part lui-même et ses futurs lecteurs, le traducteur, en lisant le texte à traduire, doit en déceler les subtilités sur tous les plans, esthétique, stylistique, rythmique et rhétorique. II lui appartient de repérer et distinguer ambiguïtés et équivoques s'il y en a, ainsi que leur nature volontaire ou involontaire suivant qu'elles procèdent du linguistique ou du situationnel.

\section{Évolution de la réception de l'équivoque}

Au delà des définitions courantes du substantif, dont les connotations, comme celles de l'adjectif, sont plus négatives que positives, le concept de l'équivoque s'auréolait d'une valeur positive jusqu'au $\mathrm{XVII}{ }^{e}$ siècle. Le double était perçu comme fécond et riche. Aussi celui qui en exploitait les possibilités et maniait la dualité avec brio faisait-il la preuve de son intelligence, ce qui lui valait l'admiration d'un auditoire conquis. Mais comme toute arme, l'équivoque est susceptible de servir à des fins éthiques ou non. Par un revirement de la pensée, le $\mathrm{XVII}$ e siècle va oublier la richesse du double pour en retenir le côté sombre et dénoncer la duplicité potentielle du beau parleur. II s'agira désormais de privilégier clarté et univocité. Ainsi, après avoir été cultivée, l'équivoque sera stigmatisée par Boileau, bientôt rejoint par ses contemporains dans toute l'Europe. Dans la célèbre satire XII, il la dénonce comme instigatrice du péché originel puis la rend responsable du schisme qui aboutit à la Réforme. La réévaluation des valeurs se manifestant dans le rapport au discours affecte l'ensemble de la société. 
On reconnaît aujourd'hui encore quelques vertus à l'équivoque, habile moyen de contourner la censure morale ou politique pour ceux qui cherchent à s'exprimer dans des pays privés de liberté d'expression. Le recours à cette forme d'expression est alors perçu non comme une manifestation de duplicité mais comme un acte de prudence. L'emploi de l'équivoque devient une habile stratégie qui dissimule derrière le sens licite, celui illicite susceptible de mettre le premier en danger. Toutefois, la défiance perdure jusqu'à notre époque éprise de traçabilité et de transparence et donc prompte à stigmatiser l'équivoque ou à passer à côté, la confondant avec une ambiguïté, comme le suggèrent les réactions de quelques traducteurs ayant eu la gentillesse d'accepter de répondre à une question envoyée par courriel sur la liste de diffusion de l'ATLF. ${ }^{7}$

\section{Étude de cas}

II s'agissait, hors contexte, sinon celui du message qui présentait les choses de manière ludique, d'essayer de traduire ou du moins de donner un sens aux phrases suivantes, inventées pour la circonstance mais que chacun pourrait un jour avoir entendues ou lues dans un dialogue: "J'ai bien roulé. Et je peux jurer que je n'étais pas à 90." Le lecteur de cet article est sur ses gardes et s'attend à l'équivoque dans la phrase. Pourtant, ce n'est pas elle qui a retenu l'attention des vingt-cinq répondants. Cinq ont déclaré comprendre spontanément qu'il s'agissait d'un automobiliste roulant à une vitesse inférieure à $90 \mathrm{~km} / \mathrm{h}$ tandis que neuf pensaient qu'il s'agissait d'un automobiliste roulant à une vitesse supérieure. Ces quatorze répondants ne donnaient pas le même sens à la phrase mais ont commencé par n'en voir qu'un. Huit ont dénoncé l'ambiguïté de la phrase tandis que deux cherchaient des compléments possibles au verbe rouler et qu'un autre l'interprétait en lui attribuant un sens métaphorique ${ }^{8}$. Le côté artificiel de la phrase et sa relative maladresse n'ont pas échappé et suscité des commentaires très justes. Certains ont essayé d'imaginer un contexte la rendant possible, évoqué la nécessité d'obtenir des informations sur le personnage la prononçant, ou d'entendre l'intonation et de savoir à qui il s'adressait et dans quel but, afin de pouvoir traduire dans un sens ou dans l'autre.

Il était effectivement difficile, sans aucune autre information, d'imaginer que l'ambiguïté, bien réelle, relevait d'une équivoque volontaire et qu'il convenait donc de la traduire. Avec un contexte plus large, l'équivoque situationnelle aurait été perçue. Dans une fiction, l'auteur, qui déciderait d'user de ce moyen, peut vouloir faire dire une chose par un de ses

\footnotetext{
${ }^{7}$ Association des Traducteurs Littéraires de France.

8 II a bien roulé sa bosse. II a vécu à 100 à l'heure.
} 
personnages à un autre, tout en souhaitant en faire comprendre une autre à un second (destinataire intradiégétique) ou au lecteur (destinataire extradiégétique). D’ailleurs les traducteurs qui se sont penchés sur la question ont bien imaginé un conducteur venant de se faire arrêter qui tente de convaincre l'agent qu'il n'a pas enfreint la loi et roulait donc à moins de $90 \mathrm{~km} / \mathrm{h}$. II suffisait d'ajouter un passager, ou un lecteur placé dans la connivence par le contexte, et qui sait que la voiture allait à plus de $90 \mathrm{~km} / \mathrm{h}$, pour apprécier le double sens de la phrase et en sourire. L'équivoque ainsi mise par un auteur dans la bouche d'un de ses personnages lui permet également de le caractériser. La présence d'un second personnage importe peu, ce qui compte, c'est l'exploitation du décalage entre les deux niveaux de narration pour créer une équivoque situationnelle ou encore contextuelle. La réussite du procédé dépend de la capacité des destinataires de ce fragment de discours imaginaire à saisir à la fois les deux sens. La compréhension du texte ne requiert pas de choisir mais d'être capable d'appréhender simultanément les deux sens. C'est en quelque sorte de leur conflagration que naît le sens véritable ou l'émotion visée par l'emploi de cet artifice destiné à bousculer la logique et à amener la pensée à une remise en question, préliminaire salutaire à l'acceptation de l'altérité.

La traduction de cette équivoque, aussi "parfaite" soit-elle - dans quelque langue que ce soit - ne pourrait être comprise par des destinataires ignorant tout de l'existence de limitations de vitesse. Si l'on voulait la traduire vers l'anglais ${ }^{9}$, il serait assez facile de tourner la difficulté constituée par le fait que l'unité utilisée n'est pas forcément la même et que la limite de vitesse varie d'un pays (ou Etat des Etats-Unis) à l'autre.

Par sa densité, l'équivoque sollicite le bagage cognitif des lecteurs en les invitant à participer à la construction du sens. Le traducteur se doit d'être l'un des meilleurs lecteurs du texte à traduire. Cet archilecteur (Plassard, 2007 : 269) repère les éventuelles erreurs inexactitudes ou coquilles et peut-être équivoques - dont il doit s'assurer qu'elles ne sont pas introduites par sa lecture mais que celle-ci se contente de les détecter. Ainsi, cette phrase dans un guide touristique sur l'Irlande écrit en anglais-: "Another, built between Cashel and Rosmuck as a famine relief measure using turf and grass, is Bóthar na Scrathóg, the road of the top-sods ${ }^{10 "}$. Elle a amené le traducteur à se demander s'il fallait comprendre, et donc traduire, une intentionnalité peut-être malveillante dans l'emploi de sod en anglais. C'est à la fois le matériau dont fut construite cette route (sod, la motte de gazon) et un terme argotique, injurieux qui ici pourrait évoquer avec mépris les paysans qui l'ont construite. La

\footnotetext{
${ }^{9}$ Une possibilité parmi sans doute beaucoup d'autres serait :

The drive was fine. And I can guarantee I was not at the speed limit.

${ }^{10}$ Mes italiques.
} 
présence du nom de la route en gaélique permettait de vérifier l'absence d'équivoque dans cette langue. Une traduction univoque, descriptive, a été proposée en français. Elle ignore peut-être, mais en tout cas n'ajoute pas, l'éventuel double entendre perceptible en anglais.

Si le traducteur avait dû le conserver, il lui aurait été difficile de retrouver en français un terme polysémique permettant de rendre l'équivoque par une équivoque. Quand le double sens est rétif au passage d'une langue à l'autre, le traducteur peut faire appel à la stratégie de compensation et créer une autre équivoque qu'il introduira ailleurs dans le texte. II y a dans ce cas une dissociation sens/forme ponctuelle, mais la forme momentanément perdue sera récupérée ailleurs. Dans sa globalité, le texte traduit conservera donc les qualités de l'original. Les équivoques initialement présentes dans le texte, introduites par son auteur ou scripteur seront, selon les possibilités offertes par la langue traduisante restituées ou recrées par d'autres moyens, avec un éventuel déplacement. Dans un autre guide, cette fois-ci sur I'Indonésie, le rédacteur avait joué avec la phrase "Life is a bitch", la transformant en "Life is a beach at the Togean island." Difficile de susciter chez le lecteur en traduction française la surprise et l'amusement qui sont ceux du lecteur anglophone à la vue du mot beach alors qu'il attendait bitch, dont les prononciations, sans être rigoureusement identiques puisque le son i de beach est long et celui de bitch court, sont assez proches pour que l'on puisse parler de quasi-homophonie. Peut-être une traduction comme "Plus belle la vie, sur l'île T..." qui fait référence à un feuilleton populaire (sur France 3 depuis 2007) produirait-elle le même résultat par un autre moyen. Parodique et ironique à la fois, la citation du titre du feuilleton en français répond à l'emploi du cliché en anglais. L'effet de surprise résulte également de la frustration d'une attente opérée par un déplacement. Mais cette fois-ci, il ne porte plus sur un mot mais se situe littéralement dans l'espace, puisque du Marseille qui sert de toile de fond à la fiction, le lecteur du guide de voyages est transporté en Indonésie.

\section{Les équivoques involontaires nées de la rencontre des cultures}

On l'a vu, ces équivoques non intentionnelles jaillissent du fait du changement de destinataire qui se produit quand il y a traduction. Le traducteur n'est pas seulement celui qui fait passer le texte d'une langue dans une autre. II est aussi (surtout ?) celui qui, ce faisant, le rend intelligible là où les différences de codes culturels ou de mode de vie, liées peut-être au milieu ou à la géographie, interdiraient la compréhension du vouloir dire de l'auteur de la langue de départ, même, si c'était possible, parfaitement traduit sur le plan linguistique, mais sans aucune médiation culturelle.

Ainsi la traduction des couleurs peut-elle être source d'équivoques, puisqu'on le sait, le spectre chromatique n'est pas découpé de la même façon dans différentes aires linguistiques, ce qui entraîne l'existence de zones mal définies où l'on hésite sur le nom de la 
couleur $^{11}$. D'ailleurs les différences de perception rendues manifestes par les termes employés dans différentes langues existent aussi entre deux locuteurs appartenant à un même groupe linguistique ou culturel. Non seulement la valeur dénotative des noms de couleur ne fait pas l'objet d'un consensus - qui n'a jamais hésité entre vert et bleu, pour finalement transiger sur turquoise ? - mais leurs connotations sont susceptibles de varier dans le temps et l'espace. Couleur du passage, le blanc fut longtemps celle du deuil en France, avant de devenir celle du mariage. Quand au noir, qui remplaça le blanc aux funérailles, il évoque aujourd'hui également le luxe, la qualité et la beauté mais plus nécessairement la tristesse. À chaque fois, les traducteurs devront trouver des solutions correspondant au type de texte et à sa fonction.

\section{De la traduction à la médiation culturelle}

S'il veut transmettre à la fois la couleur et ses connotations dans une culture où cellesci seraient tout autres, le traducteur doit faire œuvre de médiation culturelle. II dispose pour ce faire de plusieurs moyens, de la $\mathrm{NdT}$ en bas de page à des considérations détaillées en préface ou postface si la traduction relève de textes universitaires ou érudits justifiant ce type de paratexte. À défaut, il inclura une explication discrètement dissimulée dans le texte pour ne pas en interrompre la lecture. On peut alors imaginer une phrase qui contienne à la fois les sens connus des cultures d'arrivée et de départ. À la fois sourcière et cibliste, elle amènerait le lecteur à s'interroger. Partant de ce qu'il connaît, ses propres référents culturels, il en viendrait à envisager une nouvelle possibilité suscitée par la lecture de la traduction de ce texte originaire d'un ailleurs géographique, culturel ou temporel. La pratique quotidienne de la traduction de toutes sortes de textes fournit parfois de telles occasions.

Dans son rôle de médiateur, le traducteur veille aussi à ne pas susciter d'équivoque culturelle. Les mots, lus ou entendus, évoquent des représentations mentales correspondant à l'expérience de celui qui les perçoit. Mais bien souvent des mots faciles à traduire correspondent à des référents qui ne sont pas les mêmes dans les deux systèmes culturels. Les objets les plus quotidiens se métamorphosent au passage des frontières. II en est ainsi du signifiant pain, dont les traductions dans diverses langues renvoient à des formes, couleurs, saveurs et textures bien particulières, donc à une multitude de signifiés. Pour lever l'équivoque résultant de la non-correspondance des catégories du réel, il suffit parfois

\footnotetext{
11 Pour approfondir ce sujet, on se reportera aux travaux de Ph. Rapatel (Clermont 2) qui a donné une communication (non publiée à ce jour) intitulée "Essai de couleur n'est pas épreuve - ni proof - et encore moins colour test" au congrès de la SAES à Bordeaux en 2009, de Annie Mollard-Desfour, linguiste lexicographe et Jocelyne Fenandez-Vest linguiste et traductrice, qui sont intervenues lors de la journée de Printemps de l'Association Atlas (Assise de la Traduction Littéraire en Arles) consacrée à la traduction de la couleur en 2009.
} 
d'importer le mot de la langue de départ en fournissant une brève explication, en note ou dans le corps du texte. Toutefois, les choses sont parfois plus complexes

On sait qu'en France le premier étage d'un immeuble est au dessus du rez-dechaussée. II faut donc penser à ajouter ou retrancher un étage si l'on traduit de ou vers l'anglais américain ${ }^{12}$. Dans un guide touristique où le texte a une fonction informative, il faut envoyer les lecteurs au bon étage. Il est important qu'il n'y ait aucune équivoque possible. En revanche, dans un texte où l'élément signifiant est la valeur symbolique du chiffre, ce qui est envisageable dans un texte de fiction, littéraire ou non, le modifier pour renvoyer à sa valeur référentielle serait un non-sens. Combien de fois, en voyage dans un autre environnement culturel, même en parlant relativement bien la langue étrangère, s'aperçoiton que le signifié, caché par le signifiant, est tout autre que celui qu'on imaginait. Tout francophone ayant commandé un café, en anglais en Angleterre, en espagnol en Espagne, aura été surpris de constater que le breuvage arrivant dans une tasse trop grande n'avait rien à voir avec son attente. Ces exemples empruntés au quotidien, auxquels on pourrait ajouter la consigne donnée aux traducteurs travaillant pour les collections Harlequin d'adapter ce que mangent les personnages au goût des lecteurs français pour ne pas détruire l'effet romantique d'un dîner en amoureux par la mention de plats inappropriés à la circonstance, montrent bien que pour les traducteurs, l'équivoque déborde largement des cas où elle est la manifestation d'une création auctoriale portant sur le matériau qu'est la langue.

Travaillant donc en contact quasi permanent, sinon avec l'équivoque, du moins avec le risque d'équivoque, le traducteur, selon les cas, l'apprivoise et s'en sert ou au contraire veille à l'éviter. II est donc dans une position délicate où son art et sa compétence sont faciles à mettre en cause. Dans un article assez ancien, on peut lire: "C'est dans un piège d'équivoque que l'infortuné traducteur est tombé, en rendant sur une affiche : Support Expo 67, par 'Supportez l'Expo 67' (Kokas, 1969: 97)." L'auteur s'appuie sur un exemple classique de paronymie interlinguistique et estime que la ressemblance entre les mots anglais et français a amené le traducteur à écrire un contre-sens, puisque supporter en français ne veut pas dire soutenir mais plutôt tolérer. C'est possible, mais un professionnel aurait-il vraiment commis cette erreur de débutant? Son choix ne serait-il pas délibéré, fondé sur une plus grande liberté dans l'utilisation de la langue que celle de son critique? D'ailleurs, l'erreur signalée en 1969 peut en 2009 surprendre de nombreux locuteurs du français, habitués de nos jours à entendre les commentateurs sportifs parler de supporters qui

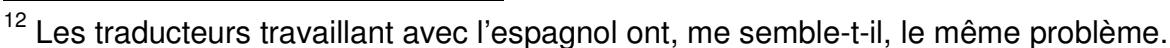


supportent leur équipe. Déjà à l'époque, la formulation ainsi mise en cause, si elle restait fautive comme le suggère la remarque, était passée dans la langue pour une grande partie des francophones. Ce sens est attesté par le TLF qui cite en exemple une phrase écrite en 1965 et tirée du journal La Croix, qui n'a jamais revendiqué une utilisation progressiste de la langue. Dans ce cas, l'équivoque ici stigmatisée n'est donc pas une audace du traducteur, comme la conclusion de l'article le porte à croire. Faut-il en conclure que l'auteur de l'article exprime par cette critique son conservatisme linguistique ? Peut-être, mais comme il précise qu'il vit à Montréal, il vaut mieux envisager qu'il est québécois. Dans ce cas, il serait malvenu de la part d'un Français de lire un conservatisme linguistique là où il convient de voir une résistance à la contamination de l'anglais. L'équivoque, si équivoque il y a, n'est pas celle que dénonce l'article. En l'absence d'information complémentaire, il convient de comprendre (et le cas échéant de traduire) sa remarque sans trancher, en laissant coexister, en suggérant même - pour un lectorat qui n'aurait pas les données culturelles - les deux possibilités d'interprétation.

\section{Équivoque et traductologie}

Ainsi comprise et utilisée, l'équivoque offre un concept au potentiel riche pour le traducteur qui œuvre à transmettre un texte ${ }^{13}$, indissociable de la culture dans laquelle il a été produit, à des lecteurs qui, le plus souvent ne parlent pas la même langue ${ }^{14}$, et surtout n'ont pas les mêmes référents culturels et pas nécessairement la même vision du monde.

Modalité du double, l'équivoque peut aussi se penser comme le lieu de coexistence des deux pôles perçus comme antagonistes autour desquels s'organise la réflexion sur la traduction. Souvent opposés et diversement appelés suivant les époques, ils se ramènent à l'opposition entre sourciers et ciblistes ${ }^{15}$. À l'instar du texte poétique, le texte traduit invite ses lecteurs à porter un autre regard sur le monde puisqu'il vient l'enrichir de la vision de l'altérité.

De l'équivoque-méprise, à l'équivoque-ouverture sur l'autre, il faut signaler avant de conclure un autre lien entre l'équivoque et la traduction. Le partage opéré par l'opposition entre ce qui est équivoque-polysémique et ce qui est univoque-monosémique, recoupe la distinction entre les textes littéraires et les autres, qu'il est bien difficile de désigner

\footnotetext{
$\overline{13}$ Par texte, on le rappelle, il faut comprendre tous les types de textes. Loin d'être cantonnée aux œuvres littéraires, l'équivoque "est le souci constant et peut-être central de cette énorme littérature à vocation pratique et pragmatique où la connaissance est strictement inféodée à l'action" (Cavaillé, 2004). Et l'auteur évoque la mémoire de fins politiques comme Richelieu et Mazarin à l'appui de cette affirmation.

${ }_{14}$ On voit à la télévision américaine des sous-titres "traduisant" des locuteurs britanniques.

15 Jean René Ladmiral a introduit ces néologismes aujourd'hui passés dans le langage courant, du moins chez les traducteurs, en 1983 lors d'une conférence prononcée à Londres. (Ladmiral, 1990)
} 
autrement que par la négation, comme non littéraires, sans se perdre dans de vaines tentatives de catégorisation On s'accorde à saluer la richesse potentielle de l'équivoque dans les premiers, "Plus une œuvre est riche, plus les plans s'y superposent, plus elle prête à de multiples interprétations. Son sens ne sera pas le même pour chacun des lecteurs, comme si les mots d'une œuvre revêtaient une propriété kaléidoscopique." (Delisle, 1984 : 31). "Tout grand texte d'auteur est polysémique, souvent à l'insu de l'écrivain lui-même." (Wuilmart, 2009 : 31). D'où il s'ensuit, à l'inverse, que l'équivoque est à proscrire des textes caractérisés par l'univocité du discours où, loin de représenter une source de richesse, elle devient, on l'a vu, coûteuse.

\section{Conclusion}

Ainsi, l'équivoque, capable de faire coexister deux visions différentes du monde et devenant symbole d'ouverture, n'est pas à l'origine de la méprise qui résulte au contraire de la non-rencontre de deux conceptions univoques. Non seulement la notion d'équivoque invalide la nécessité de se placer soit du côté de la traduction ethnocentrique cibliste soit de celui de la traduction éthique sourcière, mais elle invite à prendre quelques précautions avant d'affirmer que, de par leur nature univoque, les textes non littéraires présenteraient un niveau de difficulté moindre. En interpellant le lecteur, l'équivoque le sort de l'univocité de sa propre culture pour l'amener vers l'autre. En se penchant sur quelques exemples d'équivoque empruntés à des situations de la vie courante ou à des textes pragmatiques, on en vient à envisager l'équivoque comme modalité du double grâce à laquelle les antagonismes ${ }^{16}$ qui traversent la traductologie pourraient être dépassés. Car si les questions se posent effectivement, l'erreur est d'y répondre en choisissant une partie plus que l'autre. Aucune réponse univoque ne saurait être juste puisqu'elle apporterait immanquablement au projet de traduction une visée propre nécessairement étrangère à celle de l'auteur (ou scripteur ou orateur) qui, quant à lui, n'est ni sourcier ni cibliste. C'est ce qu'exprime fort justement la traductrice de Ernst Bloch expliquant que, pour traduire Le principe Espérance "[Elle a] préféré rester à mi-chemin entre ces deux antipodes, car si le texte français 'sentant la traduction' est inacceptable, une version française de Bloch qui occulterait la dimension germanique, à la fois philosophique et esthétique du penseur-styliste [lui] semble tout aussi condamnable." (Wuilmart, 2009 : 18-19).

\footnotetext{
${ }^{16}$ Lettre ou esprit, Cicéron ; équivalence formelle ou équivalence dynamique, Nida ; traduction éthique ou traduction ethnocentrique, Berman (Peeters, 1999).
} 


\section{Bibliographie}

BOQUET, Claude (2007). "Traduire les textes nobles, traduire les textes ignobles - une seule ou deux méthodes ? De Schleiermacher au XXI ${ }^{e}$ siècle". In : La traductologie dans tous ses états. Artois Presse Université, pp. 9-26

BOILEAU. Satire XII Sur l'équivoque [Consulté le 19/05/2009] <URL: http://fr.wikisource.org/wiki/Satire_XII_(Boileau)>

Cavaille, Jean-Pierre (2008). "Histoires d'équivoques". In: Les Cahiers du Centre de Recherches Historiques, 33 | 2004, [En ligne], mis en ligne le 05 septembre 2008. [Consulté le 19/01/2009].<URL : http://ccrh.revues.org/index254.html>.

DeLISLE, Jean (1984). L'Analyse du discours comme méthode de traduction Théorie et Pratique, édition de l'université d'Ottawa.

HeNRY, Jacqueline (2003). La traduction des jeux de mots. Paris: Presses Sorbonne Nouvelle.

KOKAS, Louis (1969). "Les longueurs de la traduction". In: Meta, vol 14, n² 2 , pp. 93-97. [Consulté le 19/01/2009] <URL : http://id.erudit.org/iderudit/003334ar/>.

LADMIRAL, Jean-René, (1990). "La traduction proligère - Sur le statut des textes qu'on traduit". In: Meta, vol 35, n¹, pp. 102-118. [Consulté le 19/01/2009] <URL : http://id.erudit.org/iderudit/003370ar/>.

MESSIAEN, Jean-Michel. "De la syllepse à l'équivoque : redécouverte du calembour avec Tabourot" <URL: http://jeanmichel.messiaen.free.fr/FPrecl/Tel_syllepse.pdf>

MONNIER, Marc. Tour Magne à Nîmes, tour magnamime [consulté le 08/08/2009]

<http://nemausensis.ifrance.com/nemausensis/nimes/poememonnier.htm>

PEETERS Jean (1999). La médiation de l'étranger. Une sociolinguistique de la traduction

Collection traductologie, Artois Presse Université.

PLASSARD, Freddie, (2007). Lire pour traduire. Presses Sorbonne Nouvelle.

REISS, Katarina, (1971). La critique des traductions, ses possibilités et ses limites, trad. fr. en 2002 par Catherine Bocquet, Artois Presse Université.

VeRLAINE, Paul. "Les Ingénues". In : Fêtes galantes, La Pléiade, Gallimard 1989, [consulté le 08/08/2009], <http://www.pitbook.com/textes/pdf/fetes_galantes.pdf>

VIAGGIO, Sergio (2004). Téoria general de la mediacion interlingüe, Universidad de Alicante.

WUILMART, Françoise, (2009). Ditl, article "Traduction" en cours de relecture, pp. 18-19. 